\title{
Evaluación de la actividad antifúngica de peróxidos sobre botrytis (Botrytis cinerea) en el cultivo de fresa (Fragaria vesca $L$.), bajo condiciones de campo abierto
}

\section{Evaluation of the antifungal activity of peroxides on botrytis (Botrytis cinerea) in strawberry cultivation (Fragaria vesca $L$.), under open field conditions}

Edwin Córdova Noriega ${ }^{1}$

\section{RESUMEN}

La efectividad antifúngica del peróxido de hidrógeno y del ácido peracético sobre Botrytis cinerea fue evaluada en el cultivo de fresa bajo condiciones de campo. Utilizando un diseño experimental en bloques completamente al azar, con 3 bloques y 12 tratamientos, con arreglo factorial $2 \mathrm{Ax} 3 \mathrm{Bx} 2 \mathrm{C}$ correspondiente a los compuestos peróxido de hidrógeno y ácido peracético, tres dosis de aplicación $(1.5 \mathrm{cc} / 1 ., 2.0 \mathrm{cc} / 1$ y $2.5 \mathrm{cc} / 1$.) y dos densidades de siembra (15 plantas/1.4 m2 y 11 plantas/1.4 m2). Las variables evaluadas fueron incidencia y severidad, en las etapas de floración y fructificación, realizando dichas evaluaciones cada 5 días. Las aplicaciones de los productos se realizaron con una frecuencia diaria. La interacción que mejor resultado alcanzó en fructificación fue la dosis de $2.5 \mathrm{cc} / 1$. de ácido peracético aplicado en una densidad de 11 plantas $/ 1.4 \mathrm{~m} 2$, reduciendo a $34 \%$ y $39 \%$ la incidencia y severidad respectivamente. En floración, la misma interacción alcanzo resultados de $6.4 \%$ en incidencia y $7.7 \%$ en severidad. La interacción con peróxido de hidrógeno no logro mostrar mayor efecto antifúngico en comparación con el ácido peracético; sin embargo, redujo la incidencia y severidad, tanto en floración como en fructificación.

Palabras clave: Botrytis cinerea, peróxido de hidrógeno, ácido peracético, efectividad antifúngica, fresa.

\begin{abstract}
The antifungal efficacy of hydrogen peroxide and peracetic acid on was effective in strawberry cultivation under field conditions. For this, a completely randomized experimental design is required, with 3 blocks and 12 treatments, with factorial arrangement $2 \mathrm{Ax} 3 \mathrm{Bx} 2 \mathrm{C}$ corresponding to the hydrogen peroxide and peracetic acid compounds, three application doses (1.5 cc/1., $2.0 \mathrm{cc} / 12.5 \mathrm{cc} / 1$.) And two planting densities (15 plants/1.4 m2 and 11 plants $/ 1.4 \mathrm{~m} 2$ ). The variables evaluated were incidence and severity, in the flowering and fruiting stage, variable evaluations evaluations every 5 days. Product applications are used on a daily basis. The interaction that had the best result in the fruiting stage was the dose of $2.5 \mathrm{cc} / 1$. of peracetic acid applied at a density of 11 plants/1.4 $\mathrm{m} 2$, reducing the incidence and severity by $34 \%$ and $39 \%$ respectively. In the flowering stage, the same interaction achieves results of $2.5 \%$ in incidence and $3.7 \%$ in severity. The interaction with hydrogen peroxide failed to show greater antifungal effect compared to peracetic acid; however, it reduced the incidence and severity, both in the flowering stage and in the fruiting stage.
\end{abstract}

Keywords: Botrytis cinerea, hydrogen peroxide, peracetic acid, antifungal stimulus, strawberry.

\footnotetext{
'Ingeniero Ambiental, Maestro en Ciencias, Docente de la Universidad Nacional Toribio Rodríguez de Mendoza de Amazonas, correo: manuel.reategui@hotmail.com

${ }^{2}$ Ingeniero Zootecnista, Maestro en Ciencias, Docente en la Universidad Nacional Toribio Rodríguez de Mendoza de Amazonas, correo: reiner.reategui@untrm.edu.pe.
} 


\section{I.INTRODUCCIÓN}

Según Maza (2008), la fresa pertenece al género Fragaria. Planta herbácea y perenne, que posee rizoma con tallos rastreros de los cuales emergen los estolones (Ferriol, 2010). Las principales zonas productoras de fresa en el Perú, son los valles de Huaura, Chancay, Huaral y Cañete, con rendimientos promedio de $17 \mathrm{t} /$ ha (Olivera, 2012). La enfermedad más importante en el cultivo de fresa es la pudrición por botrytis (Legard, Xiao, Mertely \& Chandler, 2001), esta limita la calidad del fruto y su valor comercial en el mercado (Gaitán, Ferrucho, y Herrera, 2014). Botrytis cinerea, forma anamórfica de Botryotinia fuckeliana (de Bary) Whetzel (Latorre \& Rioja, 2002), es el hongo causante del moho gris (Leroch et al., 2013), que viene dañando a más de 200 especies de plantas (Williamson, Tudzynski, Tudzynski, \& van Kan, 2007; Choquer et al., 2007). Produce la descomposición y muerte de los tejidos mediante la producción de toxinas, enzimas, entre otros (Williamson et al., 2007). Los frutos infectados se vuelven suaves formando en su superficie filamentos de color gris (Agrios, 2005). Las flores enfermas muestran heridas color café en los pétalos, en el receptáculo y en los sépalos (Koike y Bolda, 2016).

El ácido peracético (C2H4O3) es un desinfectante de amplio espectro con efectividad en hongos (Kitis, 2004). Usado en el control de enfermedades causadas por mohos, no deja residuos que afectan la calidad fisicoquímica y nutricional de las fresas (Nicolau-Lapeña et. al., 2019), siendo aceptado para su uso en la producción orgánica (Kyanko, Russo, Fernández, y Pose, 2010). Asimismo, el peróxido de hidrógeno (H2O2) es un compuesto con efecto antimicrobiano y poder oxidante (Ismail, Khandaker, Mat \& Boyce, 2015) siendo uno de los principales agentes desinfectantes alternativos de mayor importancia en los últimos años (Ölmez \& Kretzschmar, 2009). Es aplicado con éxito para la desinfección de frutas y verduras (Cerioni, Lazarte, Villegas, Rodríguez-Montelongo \& Volentini, 2013), induciendo resistencia contra patógenos (Bozsó et. al., 2005), dentro de los cuales se encuentra B. cinerea (Hafez, 2010).

Por lo anterior, el objetivo del presente estudio consistió en evaluar el efecto antifúngico de los peróxidos, relacionados con la selección de la dosis, densidad e interacción de mayor respuesta sobre botrytis (Botrytis cinerea) en el cultivo de fresa (Fragaria vesca L.), bajo condiciones de campo abierto.

\section{MATERIALES Y MÉTODOS}

El ensayo se instaló en el distrito de Huancas, Chachapoyas - Amazonas. Geográficamente la parcela experimental se ubicó a $6^{\circ} 10^{\prime} 0.28^{\prime \prime}$ de latitud sur y $77^{\circ} 51^{\prime} 56.07^{\prime \prime}$ de longitud oeste, a una altitud de
$2600 \mathrm{msnm}$. Se utilizó un diseño de bloques completos al azar (DBCA), con arreglo factorial: 2A (Producto) x 3B (Dosis) x 2C (Densidad), el cual constó de 12 tratamientos, y 3 repeticiones. Siendo los Factores en estudio: Factor A: peróxido de hidrógeno y Ácido peracético, factor B: 1.5 cc/l., 2.0 cc/l. y $2.5 \mathrm{cc} / 1$., factor C: 15 plantas $/ 1.4 \mathrm{~m} 2$ y 11 plantas $/ 1.4 \mathrm{~m} 2$. Las variables fueron incidencia $y$ severidad en flores y frutos. Para calcular incidencia se utilizó la fórmula propuesta por Pardo et al. (2017):

INCIDENCIA $(\%)=\frac{\text { Número de individuos enfermos por planta }}{\text { Número total de individuos por planta }} \underset{\text { x }}{100}$;

mientras que para el cálculo de severidad en flores y frutos se utilizó la siguiente fórmula:

SEVERIDAD (\%) $=\frac{\text { Área de tejido afectado }}{\text { área total del tejido }} \times 100$,

los resultados obtenidos en el porcentaje de severidad, se transformaron a grados mediante la escala descriptiva propuesta por Gaitán et al. (2014): Tabla 1. Índice de severidad por grados

\begin{tabular}{ll}
\hline Grados & Porcentaje del Área Infectada \\
\hline 0 & Sanas \\
1 & Menos del $5 \%$ de área infectada \\
2 & 6 al $10 \%$ de área infectada \\
3 & 11 al 25\% de área infectada \\
4 & 26 al $50 \%$ de área infectada \\
5 & Más del 50\% de área infectada \\
\hline
\end{tabular}

Se realizó la primera determinación de las variables antes de iniciar con las aplicaciones de los peróxidos y las siguientes determinaciones se realizaron durante las aplicaciones de los mismos, cada 5 días, por un periodo de 30 días. Para determinar el rendimiento, Se cosecharon los frutos evaluados en incidencia y severidad y el peso de los frutos sanos se registraron, los valores fueron expresados en $\mathrm{kg} / \mathrm{ha}$. La evaluación se realizó a partir de los frutos que tenían el color característico de la variedad, entre $2 / 3$ a 3/4 de la superficie.

Para el manejo del experimento se construyeron 36 unidades experimentales, cada una constituida por una extensión de $1.4 \mathrm{~m}$ de ancho por $1.0 \mathrm{~m}$ de largo. Se removió el suelo de cada unidad experimental y se trazaron surcos verticales con sus respectivas medidas. Para la siembra se usó estolones de fresa, adquiridas en el distrito de Huaral. Los mismos fueron desinfectados con ENLACE 50 PM, a una dosis de $200 \mathrm{ml} /$ cil.. No se usó sombra alguna para la protección de las plantas. Se aplicó riego por gravedad, el cual fue efectuado de acuerdo al clima de la zona y a la humedad del suelo. Las aplicaciones se realizaron por aspersión a partir de los tres meses y medio después de la siembra, con frecuencia diaria, y por un periodo de 30 días. Las dosis de aplicación se obtuvieron con pruebas preliminares en plantas de fresa de la misma variedad.

Previo al análisis de varianza, las variables en 
porcentajes se transformaron con la función raíz acuadrda.

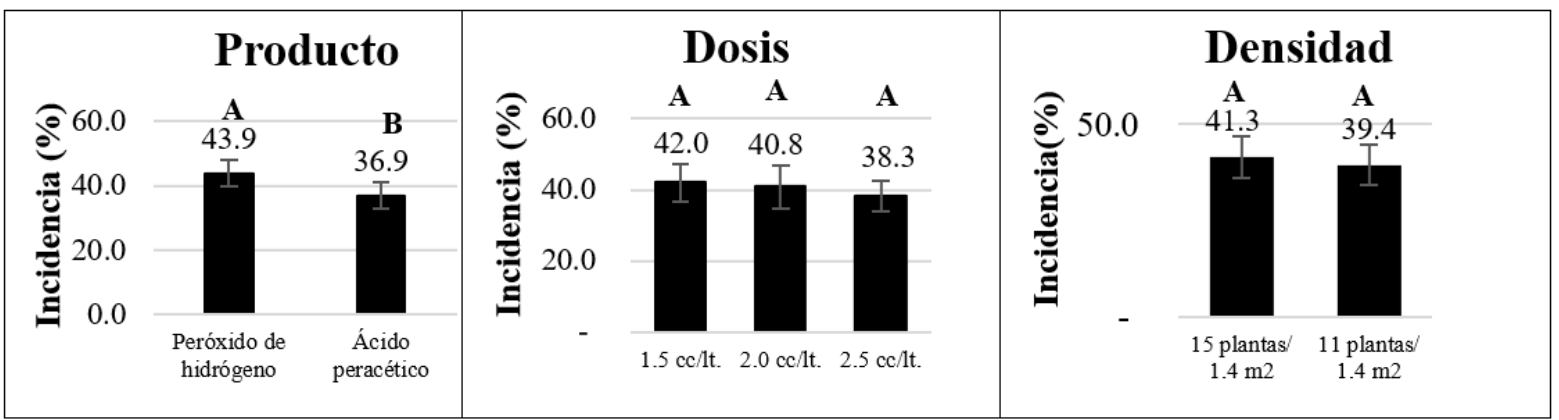

Figura 1. Incidencia producida por B. cinerea, en fructificación en función a producto, dosis y densidad.

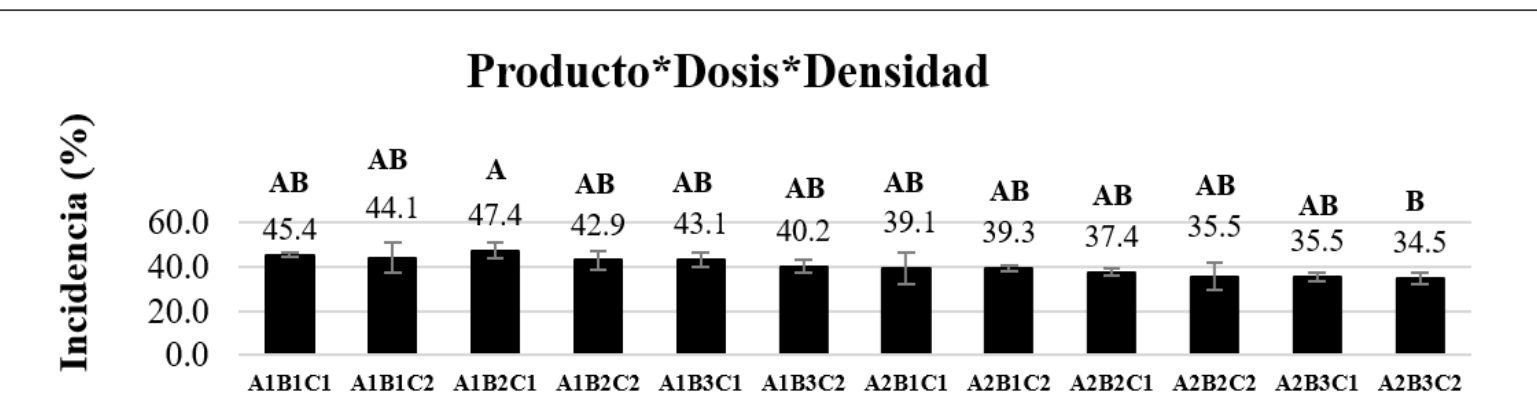

Figura 2. Incidencia producida por B. cinerea, en fructificación en función a la interacción de los factores.

Severidad en fructificación

El efecto del ácido peracético fue mayor al del peróxido de hidrógeno, presentando grado 4 de severidad. Asimismo, la dosis de $2.5 \mathrm{cc} / \mathrm{l}$. presentó mayor efecto sobre las demás, las tres dosis presentaron grado 4 de severidad (Figura 3). Por otro lado, la densidad de 11 plantas/1.4 m2 con $43.4 \%$ presentó mejor respuesta, presentando grado 4 de severidad (Figura 3). En las interacciones, se observó una mejor respuesta en $\mathrm{A} 2 \mathrm{~B} 3 \mathrm{C} 2$ con $39.2 \%$, mientras que $\mathrm{A} 1 \mathrm{~B} 1 \mathrm{C} 1$ presento mayor severidad con un $52.9 \%$, presentando grado 4 de severidad. (Figura 4).

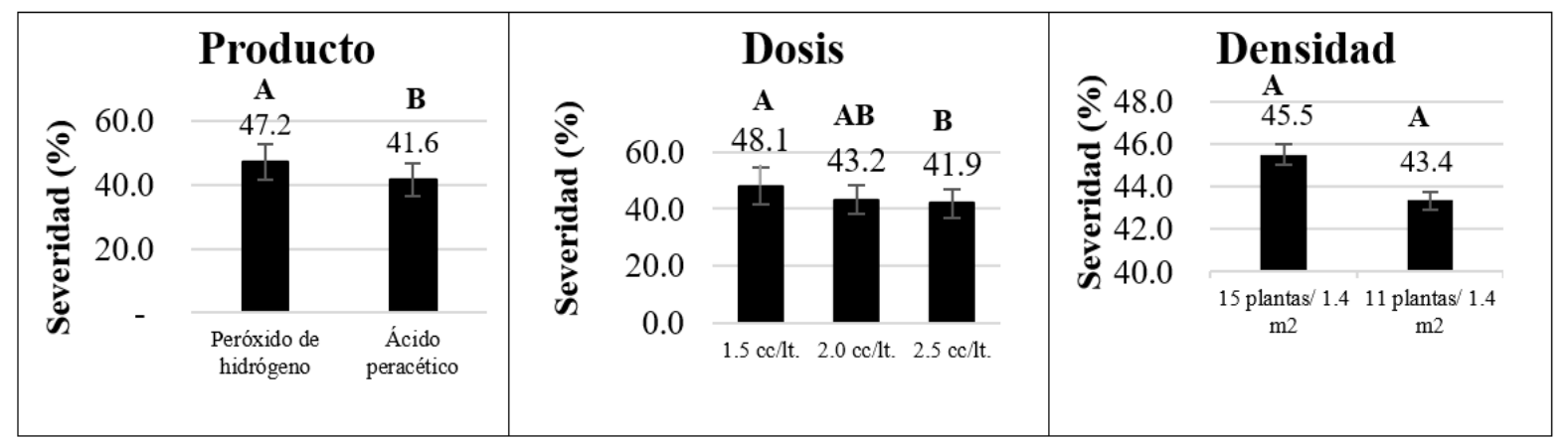

Figura 3. Severidad producida por B. cinerea, en fructificación en función a producto, dosis y densidad.

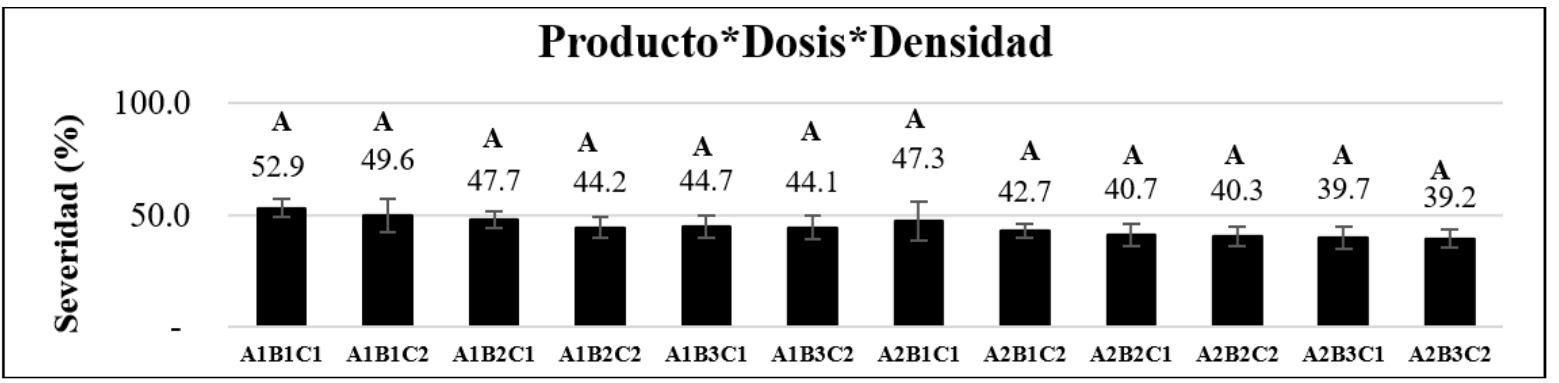

Figura 4. Severidad producida por B. cinerea, en fructificación en función a la interacción de los factores. 


\section{Incidencia en floración}

El peróxido ácido peracético fue el que menor incidencia presentó con un valor de $8.4 \%$. Asimismo, en las dosis se observa que $2.5 \mathrm{cc} / \mathrm{l}$. presento mayor efecto en incidencia con un valor de $10.1 \%$ (Figura 5). La densidad de 11 plantas/1.4 m2 con un valor de $10.3 \%$, fue superior al de 15 plantas/1.4 m2 que presento un porcentaje de $11.8 \%$, no encontrándose diferencias significativas entre ellas (Figura 5). La interacción, A2B3C2 alcanzo mayor efecto en incidencia con $6.4 \%$, mientras que la interacción que demostró tener menos efecto fue A1B1C2 con $14.7 \%$ (Figura 6).

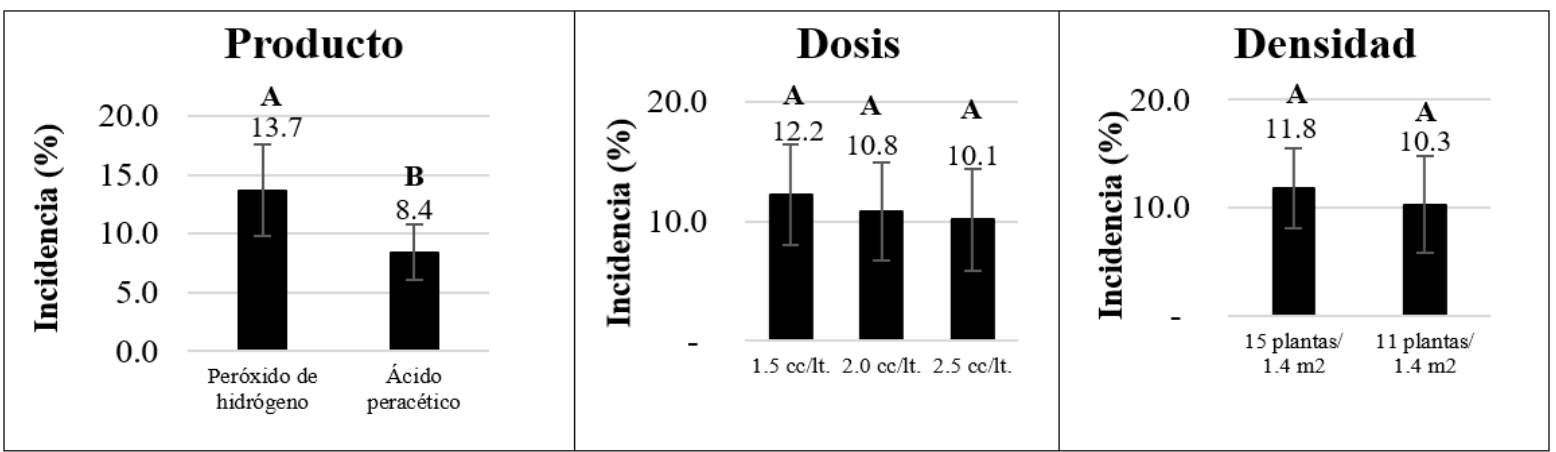

Figura 5. Incidencia producida por B. cinerea, en floración en función a producto, dosis y densidad.

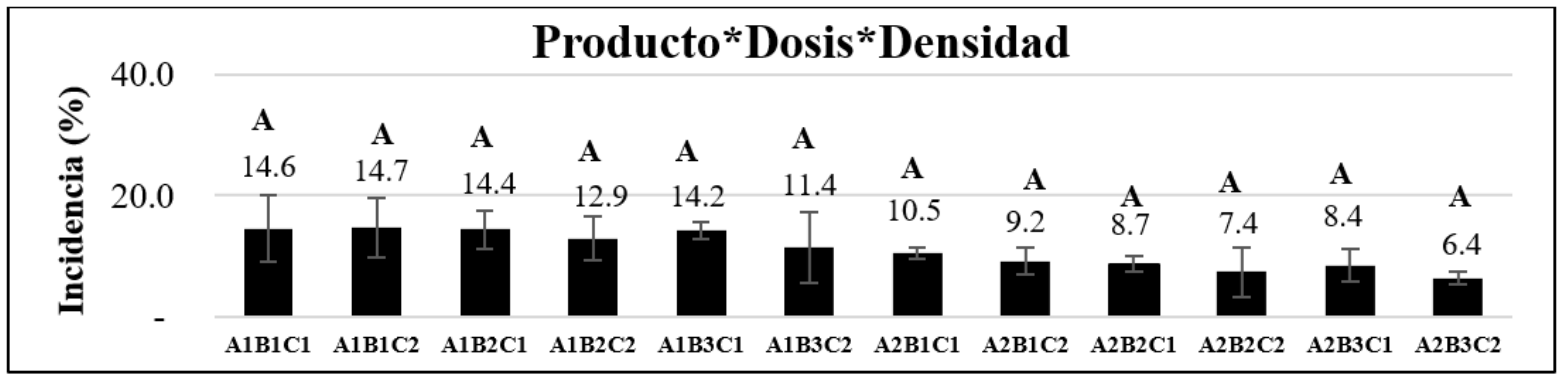

Figura 6. Incidencia producida por B. cinerea, en floración en función a la interacción de los factores.

\section{Severidad en floración}

El ácido peracético con $10.1 \%$ presentó mayor efecto en comparación al peróxido de hidrógeno con un $10.5 \%$, ambos con grados 2 de severidad (Figura 7). Asimismo, la dosis $2.5 \mathrm{cc} / \mathrm{l}$. con $9.1 \%$ presentó mayor efecto sobre las demás (Figura 7). También la densidad de 11 plantas/1.4 $\mathrm{m} 2$ con un $9.7 \%$ obtuvo mayor efecto sobre 15 plantas/ $1.4 \mathrm{~m} 2$, con un $10.9 \%$. Ambas con grado 2 de severidad, (Figura 7). En ese contexto, la interacción que mayor efecto presentó fue $\mathrm{A} 2 \mathrm{~B} 3 \mathrm{C} 2$ con $7.7 \%$ (grado 2 de severidad), mientras que $\mathrm{A} 2 \mathrm{~B} 1 \mathrm{C} 1$ presentó $13.1 \%$ (grado 3 de severidad) (Figura 8)..

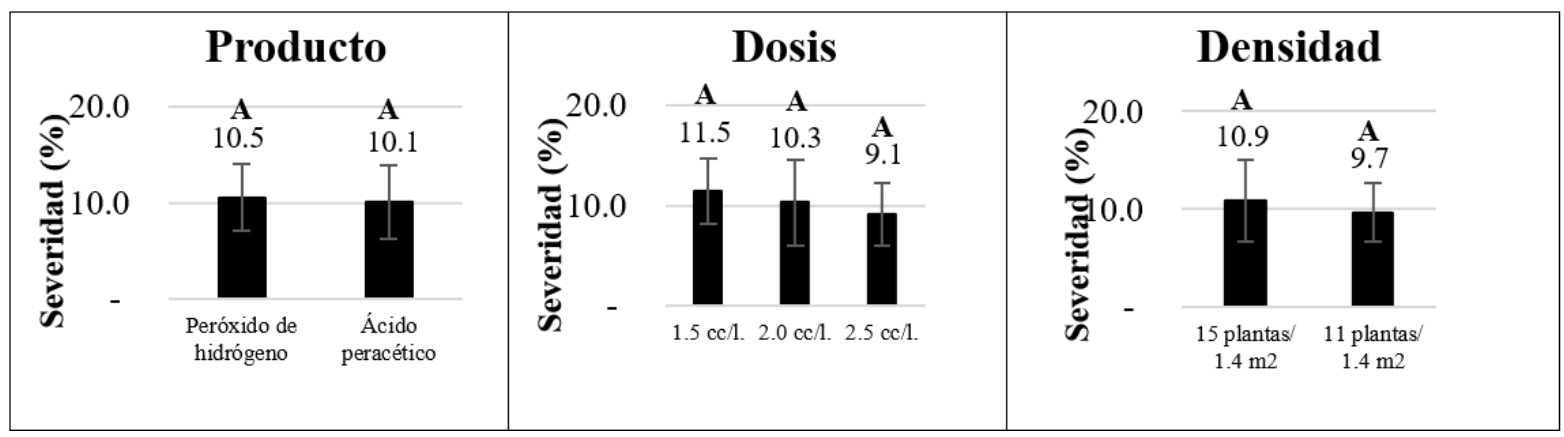

Figura 7. Severidad producida por B. cinerea, en floración en función a producto, dosis y densidad. 


\section{Producto*Dosis*Densidad}

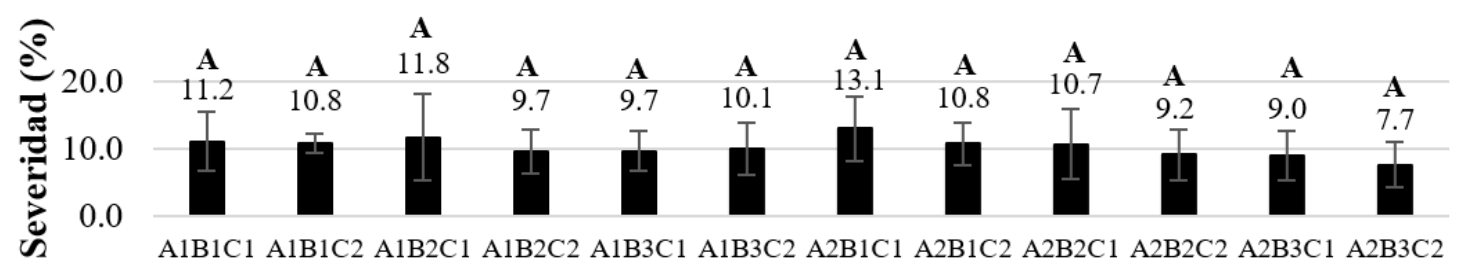

Figura 8. Severidad producida por B. cinerea, en floración en función a la interacción de los factores.

Evaluación de incidencia y severidad en frutos antes de aplicar peróxidos

Los resultados mostrados fueron recopilados 05 (cinco) días antes de realizar la primera aplicación de los peróxidos. Las variables estudiadas variaron de acuerdo a la densidad de siembra, registrando una incidencia de $44.4 \%$ para 15 plantas $/ 1.4 \mathrm{~m} 2$ y un $43.2 \%$ para 11 plantas $/ 1.4 \mathrm{~m} 2$. En cuanto a la severidad, los valores presentados fueron 51.3\% (grado 5 de severidad) para la densidad de 15 plantas $/ 1.4 \mathrm{~m} 2$ y un $51.2 \%$ (grado 5 de severidad) para la densidad de 11 plantas/1.4 m2 (Figura 19).

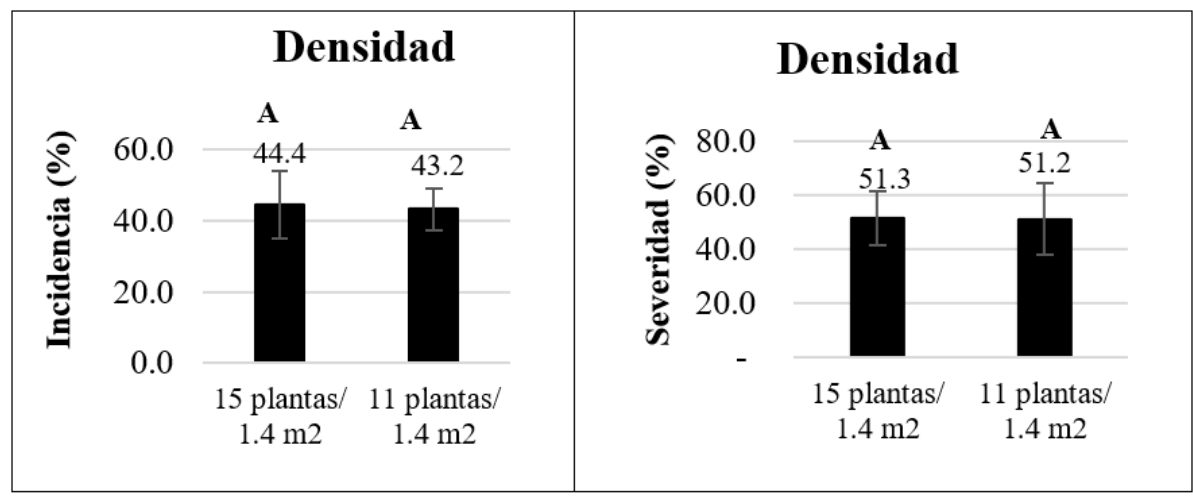

Figura 9. Incidencia y Severidad en frutos, antes de la aplicación de los peróxidos.

Evaluación de incidencia y severidad en flores antes de aplicar peróxidos

Los resultados registrados muestran valores de incidencia de $14.6 \%$ para la densidad de 15 plantas/1.4 m2 y $12.8 \%$ para la de 11 plantas $/ 1.4 \mathrm{~m} 2$.
Igualmente, en la variable severidad se observan valores de $15.3 \%$ (grado 3 de severidad) para la densidad de 15 plantas/ $1.4 \mathrm{~m} 2$ y $12.3 \%$ (grado 3 de severidad) para la densidad de 11 plantas $/ 1.4 \mathrm{~m} 2$ (Figura 10).

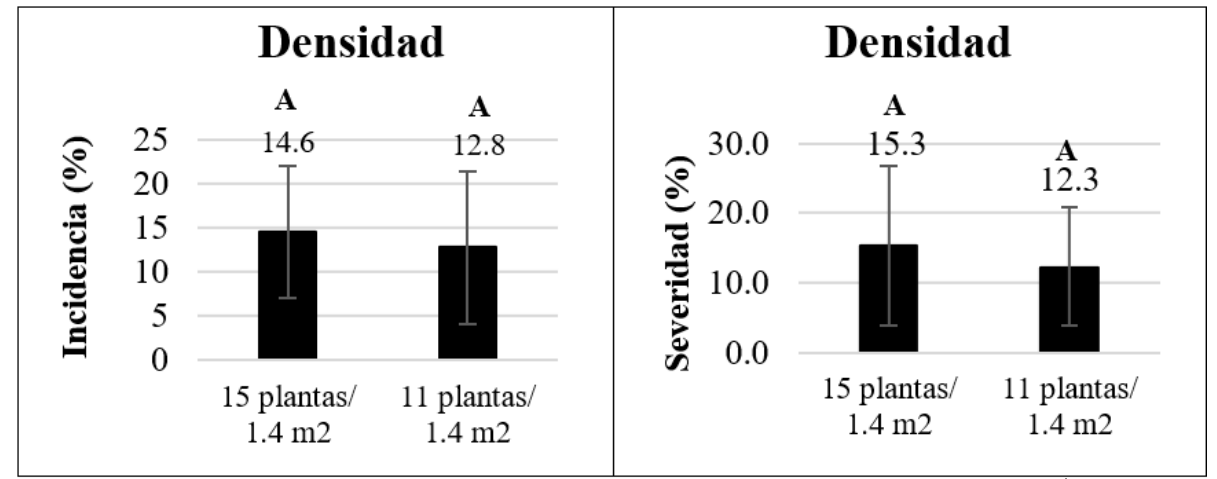

Figura 10. Incidencia y Severidad en flores, antes de la aplicación de los peróxidos.

\section{Rendimiento (kg/10 plantas)}

En la figura 11, se observa el rendimiento promedio alcanzado hasta los cuatro meses y medio después de la siembra. El ácido peracético es el peróxido que mayor respuesta alcanzó con $0.75 \mathrm{~kg} / 10$ plantas. Asimismo, en las dosis se observa un incremento del rendimiento con $2.0 \mathrm{cc} / \mathrm{l}$. alcanzando un $0.72 \mathrm{~kg} / 10$ plantas; mientras que la densidad de siembra que mejores resultados obtuvo es la de 15 plantas $/ 1.4 \mathrm{~m} 2$ con un rendimiento de $0.71 \mathrm{~kg} / 10$ plantas. La interacción $\mathrm{A} 2 \mathrm{~B} 2 \mathrm{C} 1$ presentó mayor rendimiento con $0.871 \mathrm{~kg} / 10$ plantas, seguido por las interacciones A1B3C2 y A2B1C1 con 0.753 y 0.751 $\mathrm{kg} / 10$ plantas respectivamente (figura 12 ). 


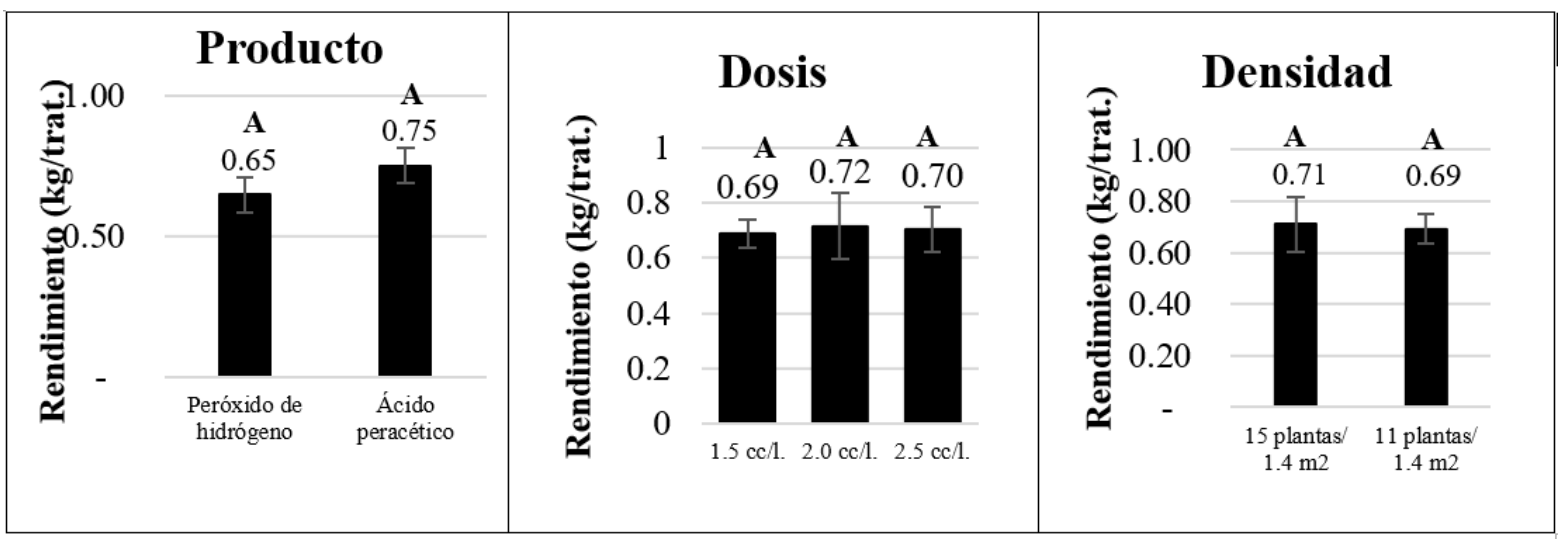

Figura 11. Rendimiento de la fresa en cada uno de los factores.

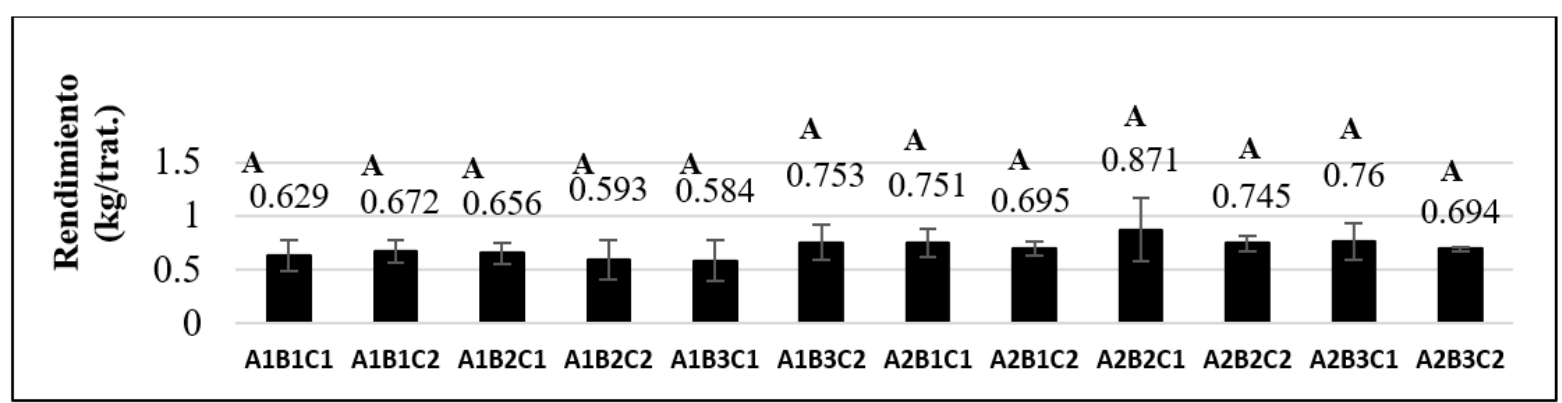

Figura 12. Rendimiento de la fresa en cada una de las interacciones.

\section{DISCUSIÓN}

La mayor parte de estudios sobre el efecto que producen los compuestos orgánicos en botrytis, se han realizado a nivel de laboratorio en etapa de poscosecha y en algunos casos en invernadero. Hasta la fecha son escasas los trabajos en condiciones no controladas. En ese sentido, en todos los tratamientos evaluados se observó infección por B. cinerea, presentando sus primeros síntomas a los tres meses después de la siembra.

La mayor efectividad del ácido peracético sobre botrytis se puede deber al amplio poder oxidante que ejerce sobre los hongos, ya que resulta de la combinación de ácido acético y peróxido de hidrógeno, concordando con Kyanko et al. (2010). Asimismo, el menor efecto del peróxido de hidrogeno sobre las variables estudiadas puede deberse al corto poder residual que tiene al entrar en contacto con el medio ambiente, durando solo unos minutos y siendo ineficiente contra infecciones de botrytis que ocurran poco tiempo después de su aplicación. Asimismo, Rebollar (2011), afirma que el peróxido de hidrógeno es más un preventivo que no ejerce mucho efecto como controlador de enfermedades.

Por otro lado, se pueda observar que la incidencia y severidad disminuye conforme se aumenta la dosis. Referente a este factor, Leggett et al. (2016) en su investigación demuestran que a concentraciones más bajas de ambos peróxidos el efecto esporicida es relativamente mucho menor. Por otro lado, CuervoUsán et al. (2014) señalan, que la eficacia de los peróxidos sobre botrytis se obtiene con dosis desde 0.128 , concluyendo que a más dosis de dichos compuestos menor será el porcentaje de enfermedad. Asimismo, al observarse que la densidad que mejores resultados alcanzo fue de 11 plantas/1.4 m2. Estos valores indicarían que, a mayor distancia entre plantas, menor será la incidencia y severidad, concordando con Agrios (2005) y Sahile et al. (2008). A mayor densidad más sombra existirá y mayor será el tiempo de la humedad en el suelo, por ende, los frutos que estén en contacto con el suelo serán más susceptibles a pudrirse e incitar el desarrollo del hongo en su superficie, corroborando lo que afirma Coscollá (1980), que en muchas enfermedades las esporas, conidios y otros para poder germinar necesitan de agua y/o de ambientes húmedos; también Davidson et al. (2007) afirma que B. cinerea se desarrolla en estaciones húmedas y cultivos de alta densidad de siembra.

mayoría de flores evaluadas no entraban en contacto con el suelo, por ende, no eran afectadas tan severamente por el hongo ni por otros patógenos que propiciaban el desarrollo del mismo.Por otro lado, en cuanto a rendimiento la mejor interacción fue de 2.0 cc/l. de ácido peracético en una densidad de 15 plantas/1.4 m2 (A2B2C1), este resultado podría deberse a que siendo el ácido peracético el peróxido que mayor efecto sobre botrytis demostró, por ende, redujo la cantidad de frutos enfermos, también la densidad de siembra en el presente estudio fue un factor muy importante, ya que según Morales (2017), la densidad de siembra para esta variedad es de 
mínimamente $26 \mathrm{~cm}$. entre planta, y por ende las dos densidades están en el rango de siembra para esta variedad. Estigarribia \& Pino (2013), señalan que mientras mayor sea la distancia de siembra, menor será la competencia entre las plantas y mayor será el rendimiento por planta.

\section{CONCLUSIONES}

El ácido peracético presentó mayor efecto en todos los tratamientos, Asimismo, la dosis $2.5 \mathrm{cc} / 1$ presentó mejores respuestas sobre botrytis, tanto en etapa de fructificación como en floración. También la densidad de siembra que presentó mejores resultados sobre botrytis fue de 11 plantas/1.4 m2. La interacción de ácido perácetico a una dosis de 2.0 cc/1. con una densidad de siembra de 15 plantas/1.4 $\mathrm{m} 2$ alcanzo un mayor rendimiento. Los mejores resultados para disminuir incidencia y severidad se obtuvieron con interacciones de $2.5 \mathrm{cc} / \mathrm{l}$. de ácido peracético aplicado en una densidad de 11 plantas $/ \mathrm{m} 2$.

\section{REFERENCIAS BIBLIOGRÁFICAS}

Agrios, G. N. (2005). Plant pathology. - 5th ed. USA: Academic press.

Bozsó, Z., Ott, P. G., Szatmari, A., Czelleng, A., Varga, G., Besenyei, E., ... \& Klement, Z. (2005). Early detection of bacterium-induced basal resistance in tobacco leaves with diaminobenzidine and dichlorofluorescein diacetate. Journal of phytopathology, 153(10), 596-607.

Cerioni, L., de los Ángeles Lazarte, M., Villegas, J. M., Rodríguez-Montelongo, L., \& Volentini, S. I. (2013). Inhibition of Penicillium expansum by an oxidative treatment. Food microbiology, 33(2), 298-301.

Choquer, M., Fournier, E., Kunz, C., Levis, C., Pradier, J. M., Simon, A., \& Viaud, M. (2007). Botrytis cinerea virulence factors: new insights into a necrotrophic and polyphageous pathogen. FEMS microbiology letters, 277(1), 1-10.

Coscollá, R. (1980). Incidencia de los factores climatológicos en la evolución de las plagas y enfermedades de las plantas. Bol. Serv. Plagas, 6(2), 123-139.

Cuervo-Usán, Y., Tornos-Mauri, P., HernándezDomínguez, J. C., Orihuela-Calvo, D., Domínguez-Hernández, M. E., \& MorenoMartínez, E. (2014). Eficacia de peróxidos en la desinfección de suelos aptos para el cultivo de fresa en el Mediterráneo. Revista fitotecnia mexicana, 37(4), 393-398.

Davidson, J. A., Pande, S., Bretag, T. W., Lindbeck, K. D., \& Krishna-Kishore, G. (2007). Biology and management of Botrytis spp. in legume crops. In Botrytis: biology, pathology and control (pp. 295-318). Springer, Dordrecht.

Estigarribia, O.S., \& Pino, P.S. (2013). Efecto de la competencia de malezas y la densidad de siembra en el rendimiento del cultivo de algodón (Gossypium hirsutum L.) var. Coodetec 405. Investigación agraria, 10(2), 21-28.

Ferriol, X. (2010). Propiedades nutritivas y otras curiosidades de la fresa. Revista Citrifrut, 27(1), 72-74.

Gaitán, J. B. M., Ferrucho, R. L., \& Herrera, J. G. Á. (2014). Efecto de dos cepas de Trichoderma en el control de Botrytis cinerea y la calidad del fruto en fresa (Fragaria sp.). Revista Colombiana de Ciencias Hortícolas, 8(1), 4456.

Gaitán, J. B. M., Ferrucho, R. L., \& Herrera, J. G. Á. (2014). Efecto de dos cepas de Trichoderma en el control de Botrytis cinerea y la calidad del fruto en fresa (Fragaria sp.). Revista Colombiana de Ciencias Hortícolas, 8(1), 4456.

Hafez, Y. (2010). Control of Botrytis cinerea by the resistance inducers benzothiadiazole (BTH) and hydrogen peroxide on white pepper fruits under postharvest storage. Acta Phytopathologica et Entomologica Hungarica, 45(1), 13-29.

Ismail, S. Z., Khandaker, M. M., Mat, N., \& Boyce, A. N. (2015). Effects of hydrogen peroxide on growth, development and quality of fruits: a review. JAgron, 14(4), 331-336.

Kitis, M. (2004). Disinfection of wastewater with peracetic acid: a review. Environment international, 30(1), 47-55.

Koike, S., \& Bolda, M. (2016). El moho gris, o Pudrición de fresa. California: Cooperative Extensión.

Kyanko, M. V., Russo, M. L., Fernández, M., \& Pose, G. (2010). Efectividad del ácido peracético sobre la reducción de la carga de esporas de mohos causantes de pudrición poscosecha de frutas y hortalizas. Información tecnológica, 21(4), 125-130.

Latorre, B.A., \& Rioja, M. E. (2002). The effect of 
temperature and relative humidity on conidial germination of Botrytis cinerea. Ciencia e Investigación Agraria, 29(2), 66-72.

Legard, D. E., Xiao, C. L., Mertely, J. C., \& Chandler, C. K. (2001). Management of botrytis fruit rot in annual winter strawberry using captan, thiram, and iprodione. Plant disease, 85(1), 31-39.

Leggett, M. J., Schwarz, J. S., Burke, P. A., McDonnell, G., Denyer, S. P., \& Maillard, J. Y. (2016). Mechanism of sporicidal activity for the synergistic combination of peracetic acid and hydrogen peroxide. Appl. Environ. Microbiol., 82(4), 1035-1039.

Leroch, M., Plesken, C., Weber, R. W., Kauff, F., Scalliet, G., \& Hahn, M. (2013). Gray mold populations in German strawberry fields are resistant to multiple fungicides and dominated by a novel clade closely related to Botrytis cinerea. Appl. Environ. Microbiol., 79(1), 159-167.

Maza, S. (2008). Estudio de fresa en el Perú y el Mundo". Ministerio de Agricultura de Perú, 1.

Morales, C. (2017). Manual de manejo agronómico de la frutilla. Santiago: Instituto de desarrollo agropecuario.

Nicolau-Lapeña, I., Abadias, M., Bobo, G., AguilóAguayo, I., Lafarga, T., \& Viñas, I. (2019). Strawberry sanitization by peracetic acid washing and its effect on fruit quality. Food microbiology, 83, 159-166.

Olivera Soto, J. (2012). Cultivo de fresa (Fragariax ananassa Duch.). Instituto Nacional de Innovación Agraria. Lima, Perú.

Ölmez, H., \& Kretzschmar, U. (2009). Potential alternative disinfection methods for organic fresh-cut industry for minimizing water consumption and environmental impact. LWT-Food Science and Technology, 42(3), 686-693.

Pardo, N. J. C., Darghan, A., Rico, M. D. S., \& Rodríguez, A. (2017). Análisis espacial de la incidencia de enfermedades en diferentes genotipos de cacao (Theobroma cacao L.) en El Yopal (Casanare), Colombia. Acta Biológica Colombiana, 22(2), 209-220.

Rebollar Alviter, A. (2011). Manejo del mildiu y el moho gris de la Zarzamora en Michoacán (No. PAFOLLETO 3535).
Sahile, S., Ahmed, S., Fininsa, C., Abang, M. M., \& Sakhuja, P. K. (2008). Survey of chocolate spot (Botrytis fabae) disease of faba bean (Vicia faba L.) and assessment of factors influencing disease epidemics in northern Ethiopia. Crop Protection, 27(11), 14571463.

Williamson, B., Tudzynski, B., Tudzynski, P., \& van Kan, J. A. (2007). Botrytis cinerea: the cause of grey mould disease. Molecular plant pathology, 8(5), 561-580. 